\title{
Rashba splitting of graphene-covered Au(111) revealed by quasiparticle interference mapping
}

\author{
Philipp Leicht, Julia Tesch, Samuel Bouvron, Felix Blumenschein, Philipp Erler, Luca Gragnaniello, and Mikhail Fonin* \\ Fachbereich Physik, Universität Konstanz, 78457 Konstanz, Germany
}

(Received 31 July 2014; revised manuscript received 29 October 2014; published 3 December 2014)

\begin{abstract}
We report on low-temperature scanning tunneling spectroscopy measurements on epitaxial graphene flakes on $\mathrm{Au}(111)$. We show that using quasiparticle interference (QPI) mapping, we can discriminate between the electronic systems of graphene and $\mathrm{Au}(111)$. Beyond the scattering vectors, which can be ascribed to the elastic scattering within each of the systems, we observe QPI features related to the scattering process between graphene states and the $\mathrm{Au}(111)$ surface state. This additional interband scattering process at the graphene/Au(111) interface allows the direct quantitative determination of the Rashba-splitting of the Au(111) surface state, which cannot be evaluated from QPI measurements on pure $\mathrm{Au}(111)$. This experiment demonstrates a unique local spectroscopic approach to investigate the Rashba-split bands at weakly interacting epitaxial graphene/substrate interfaces.
\end{abstract}

PACS number(s): 73.20.At, 68.37.Ef, 72.10.Fk, 73.22.Pr

The spin-orbit (SO) interaction in combination with broken space inversion symmetry at surfaces leads to a spin splitting of surface states in heavy metals as experimentally observed by photoemission for instance at the $\mathrm{Au}(111)$ [1-3], $\mathrm{Bi}(111)$ [4,5], and $\mathrm{Sb}(111)$ [6] surfaces. This so called Rashba-Bychkov effect [7] is further enhanced for surface alloys, such as $\mathrm{Bi} / \mathrm{Ag}(111)$ [8,9], $\mathrm{Pb} / \mathrm{Ag}(111)$ [10], and $\mathrm{Sb} / \mathrm{Ag}(111)$ [11], leading to a giant Rashba splitting. Moreover, recent studies point out the possibility of an induced extrinsic Rashba splitting in graphene on $\mathrm{Au}$ [12-14]. Compared with the intrinsic spin-orbit coupling (SOC) in graphene, which is in the range of $50 \mu \mathrm{eV}$ [15-17], the Rashba-type splitting induced by the presence of Au has recently been reported to reach $100 \mathrm{meV}$ [14].

Although the Rashba-split bands are readily visible in angle-resolved photoemission experiments [1-3], their observation at the local scale by means of scanning tunneling microscopy (STM) employing quasiparticle interference (QPI) mappings is challenging. Within such an experiment, one probes the local density of states (LDOS) oscillations generated by QPIs arising from elastic scattering between different momentum eigenstates. These standing wave patterns give rise to characteristic features in Fourier-transformed local density of states (FT-LDOS) maps, which can be understood using joint density of states (JDOS) considerations [18-20], i.e., the simple evaluation of principle scattering vectors connecting states on the constant-energy contour (CEC) of the system. However, in the case of spin-split bands only scattering between states with the same spin polarization occurs, making it impossible to reconstruct the full spin-split band structure by analysis of QPI [21-24]. In particular cases, the presence of both spin-split and spin-degenerate surface state bands allows interband transitions, which yield the information about the Rashba splitting [25]. In $\mathrm{Au}(111)$, however, only spinconserved backscattering within the surface state is observed, which masks the actual spin splitting, hence necessitating a different approach for the local observation of the Rashba splitting.

Here, we demonstrate that a direct observation of the Rashba splitting in QPI is indeed made possible using graphene. Low-temperature STM is implemented to study the

*mikhail.fonin@uni-konstanz.de quasiparticle scattering in epitaxial graphene nanoflakes on the $\mathrm{Au}(111)$ surface. Very weak interaction between graphene and $\mathrm{Au}(111)$ gives the possibility to investigate the electronic structure of each of the materials. In addition to graphenerelated and Au-related scattering, we observe QPI features due to the interband scattering between graphene and Au states, which allow for the direct quantitative determination of the spin splitting of the $\mathrm{Au}(111)$ surface state at the local scale by STM. We also comment on the possibility to observe the recently reported giant Rashba splitting of graphene [12-14] in the graphene/Au system by means of QPI.

Experiments were performed in a two-chamber ultrahigh vacuum (UHV) system (base pressure $p \leqslant 5 \times 10^{-11}$ mbar) equipped with an Omicron Cryogenic scanning tunneling microscope. All measurements were performed at $10 \mathrm{~K}$ in constant-current mode using electrochemically etched and UHV flash-annealed polycrystalline tungsten tips. $d I / d V$ maps were recorded using a lock-in amplifier with a modulation voltage of 3-4 $\mathrm{mV}_{\text {rms }}$ and a modulation frequency of $672 \mathrm{~Hz}$. The bias voltage $V$ is given with respect to the sample. $d I / d V$ maps were recorded with atomic resolution and subsequently corrected to match the graphene honeycomb lattice with the lattice parameter of $a_{G}=2.46 \AA$. The preparation of the graphene on $\mathrm{Au}(111)$ samples was performed as described elsewhere [26]. In brief, temperature-programmed growth [27] for the preparation of single-crystal graphene flakes on $\operatorname{Ir}(111)$ is used with subsequent intercalation of a nominally $50 \AA$ thick Au layer.

Figure 1(a) represents the typical surface morphology of graphene/Au(111). The intercalation of thick Au layers produces flat terraces of up to several hundred nanometers in width, which support the graphene flakes [26]. For the experiments described below we chose a graphene flake with the lateral dimensions of approximately $450 \times 140 \mathrm{~nm}^{2}$. Figure 1(b) shows the graphene flake covering the herringbone reconstructed $\mathrm{Au}(111)$ surface like a carpet. The Fast Fourier transform (FFT) of the same area is presented in Fig. 1(c), exhibiting spots related to the graphene reciprocal lattice (highlighted by a dotted hexagon) and the moiré superstructure (highlighted by a dash-dotted hexagon). The graphene atomic lattice does not feature a honeycomb appearance in the presented STM topography [Fig. 1(d)] firstly due to the presence of a large rotation angle between $\mathrm{Au}[1 \overline{10}]$ and $\mathrm{C}$ $[11 \overline{2} 0]$ of $(20.5 \pm 1.5)^{\circ}$ and the resulting moiré superstructure 

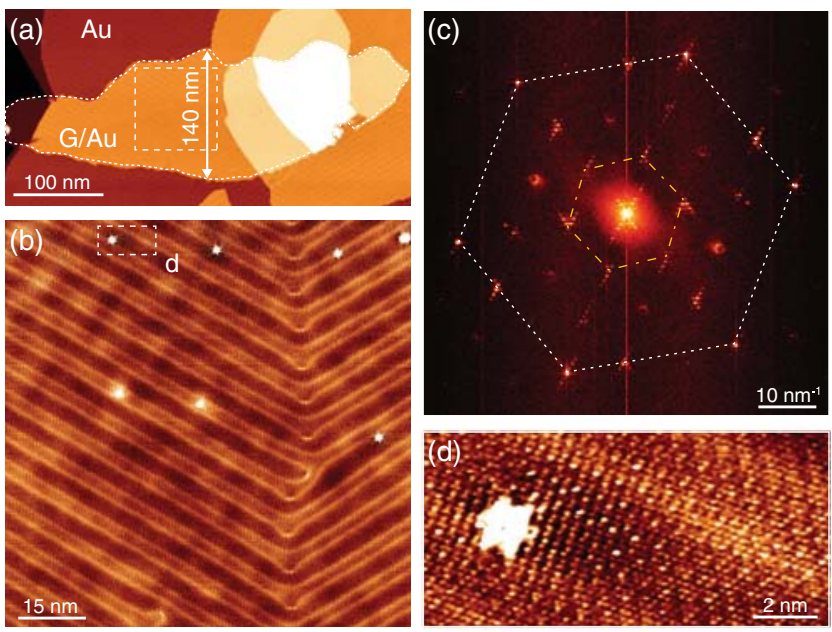

FIG. 1. (Color online) (a) Topography of the graphene flake on $\mathrm{Au}(111)$. (b) Atomic resolution of the area indicated in the overview (a). The corresponding FFT is depicted in (c) highlighting the reciprocal lattice spots (dotted hexagon) and the Moiré unit cell (dash-dotted hexagon). (d) Magnification of the area around the defect marked in (b) displaying the atomic lattice. Scanning parameters: (a) $V=100 \mathrm{mV}, I=0.5 \mathrm{nA}$; (b), (d) $V=-20 \mathrm{mV}, I=1.0 \mathrm{nA}$.

with a short periodicity of $(0.68 \pm 0.04) \mathrm{nm}$, secondly due to the LDOS modulations, which become visible also in STM topographies in the case of small bias voltages. In our previous work [26], we found that graphene on Au reveals abundant LDOS modulations especially in the vicinity of edges and defects [Fig. 1(d)] arising from QPIs, which allow accessing the graphene electronic properties by STM [20,28-30]. Rotations of the graphene sheet with respect to the substrate and the resulting moiré structure have no impact on the following results as similar effects were observed on several different flakes with various rotation angles.

We now analyze the LDOS using $d I / d V$ maps acquired with two different tip configurations, which were often obtained in the course of the experiment. Figure 2(a) shows the $d I / d V$ map acquired with tip configuration 1 at a bias voltage of $-20 \mathrm{mV}$ on the area represented in Fig. 1(b). The map shows predominantly one long wavelength modulation feature in addition to subtle modulations at the atomic scale (inset) and both of them can be clearly assigned to QPIs of graphene by analyzing their position in the FFT [Fig. 2(b)]. We focus on the ringlike features at the corners of the Brillouin zone (BZ) which can be assigned to the LDOS modulations due to the intervalley scattering in graphene [28,30], i.e., by elastic scattering between adjacent Dirac cones at $K$ and $K^{\prime}$ [Fig. 3(a)]. ${ }^{1}$ Constructive interference is found for the backscattering process, i.e., for the antiparallel orientation between $\vec{k}_{\mathrm{G}}$ and $\vec{k}_{\mathrm{G}}^{\prime}$, where $\vec{k}_{\mathrm{G}}$ and $\vec{k}_{\mathrm{G}}^{\prime}$ is measured from the $K$ and $K^{\prime}$ point of the BZ, respectively. The resulting scattering vectors $\vec{q}_{\mathrm{G} \text {,inter }}=\overrightarrow{\Gamma K}-2 \vec{k}_{\mathrm{G}}$ form six ringlike features with radius $2 k_{\mathrm{G}}$ around the corners of the $\mathrm{BZ}$ (dashed hexagon).

\footnotetext{
${ }^{1}$ For the sake of clarity we use the notation $\Gamma$ and $K\left(K^{\prime}\right)$ for the surface BZ throughout the manuscript.
}
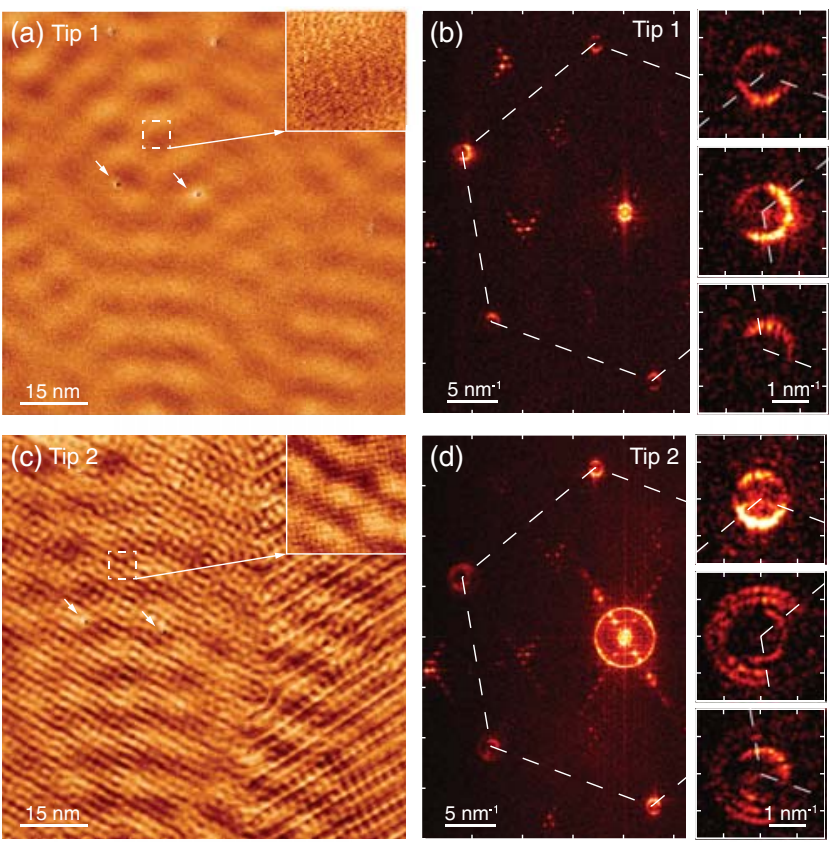

FIG. 2. (Color online) (a), (c) $d I / d V$ maps of the area of Fig. 1(b) acquired with two different tip configurations. The white arrows point at defects to show that both maps were acquired within the same region of the graphene flake. (b), (d) The corresponding FFTs are depicted including magnifications $\left(3.5 \times 3.5 \mathrm{~nm}^{-2}\right)$ of the intervalley scattering features. The dashed hexagon indicates the graphene BZ. Scanning parameters: (a) $88 \times 88 \mathrm{~nm}^{2}, V=-20 \mathrm{mV}$, $I=1.0 \mathrm{nA}$; (c) $90 \times 90 \mathrm{~nm}^{2}, V=-10 \mathrm{mV}, I=1.0 \mathrm{nA}$.

A schematic representation of the scattering vector and the expected feature in FT-LDOS is depicted in Figs. 3(b) and 3(c) (black arrow and black circle). An azimuthal variation of the intensity of the circular scattering features observed in the experiment is supposed to be related to the pseudospin texture of the quasi-freestanding graphene [29-31]. The small circular feature in the center of the FFT originates from intravalley backscattering. In freestanding monolayer graphene the intravalley backscattering is suppressed due to pseudospin conservation [29-31]; however, confinement in graphene nanoribbons may restore the intravalley feature [32].

In Fig. 2(c) the $d I / d V$ map of the same area of the flake as in Fig. 2(a) is shown acquired with the second tip configuration. Defects marked by arrows show that the imaged area is unchanged. The LDOS now exhibits additional spatial modulations similar to the backscattering of the $\mathrm{Au}$ surface state electrons [33,34]. Indeed, the FFT clearly shows an additional ringlike feature around $\vec{q}=0$ [Fig. 2(d)] assigned to the surface state of $\mathrm{Au}(111)$, which was shown to survive underneath graphene, shifted by $\simeq 100 \mathrm{meV}$ to lower binding energy and significantly contributing to the tunneling current [26]. A more subtle difference between the two maps with different tip configurations can be noticed for the features related to the intervalley scattering [magnifications in Fig. 2(d)]. For the second tip state, two ringlike features in addition to each intervalley scattering ring are observed at the corners of the BZ. Since these two additional rings were absent in the maps with the purely graphene sensitive tip 
(a)

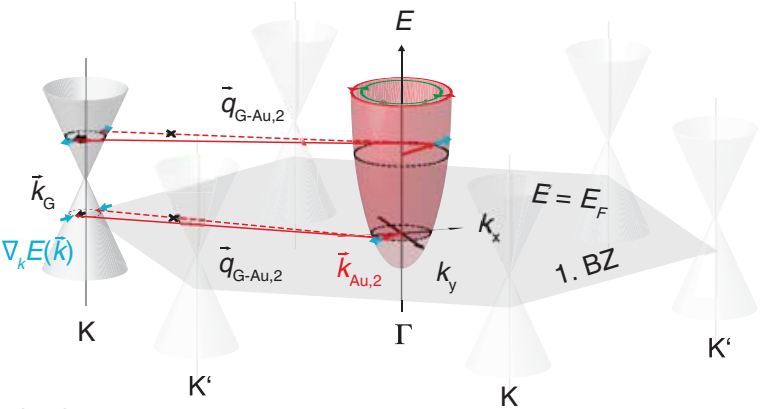

(b) CEC

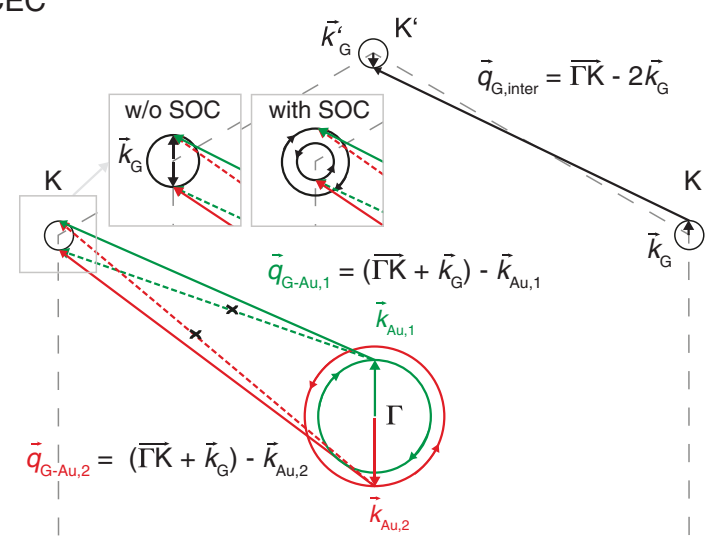

(c) FT-LDOS

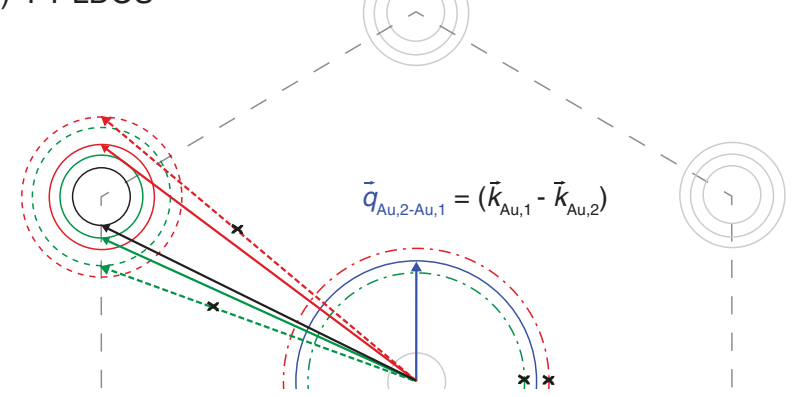

FIG. 3. (Color online) (a) Schematic representation of the possible scattering processes between one parabola of the Rashbasplit surface state and the graphene cone, corresponding to the experimental situation. Two scattering processes are depicted, one of them involving graphene states below the Dirac point, the other one involving the graphene states above the Dirac point. The reversal of the direction of $\nabla_{k} E(\vec{k})$ with respect to the $K\left(K^{\prime}\right)$ point of graphene (cyan arrows) between energies above and below the Dirac point is depicted. (b) CEC of $p$-doped graphene $\left(E_{D}=0.24 \mathrm{eV}\right)$ and $\mathrm{Au}(111)$ surface state $\left(E_{0}=-0.3 \mathrm{eV}, 396 \mathrm{meV} \AA\right)$ at an energy of $-10 \mathrm{meV}$. For the sake of clarity, scattering features are presented with twice the size with respect to graphene BZ. The insets show the situation at the $K$ point with/without a graphene SOC. (c) Corresponding FT-LDOS considering only scattering between points with antiparallel gradients $\nabla_{k} E(\vec{k})$ (gray). Experimentally observed scattering is depicted in full lines, whereas the processes which do not occur are depicted by dashed lines.

configuration, we can expect their origin to be connected to interband scattering processes involving the electronic states of $\mathrm{Au}$ and graphene. Both tip configurations were prepared reproducibly within several experiments and allow us to unambiguously discern the contribution of metal electrons and graphene electrons using QPI analysis. This is an advantage over single point scanning tunneling spectroscopy (STS) on graphene on metals, where a prevailing contribution to the tunneling current stems from surface state electrons compared to contributions from the graphene states and masks features originating from graphene [35].

For the evaluation of the additional graphene-Au scattering vectors we take into account the Rashba splitting of the $\mathrm{Au}(111)$ surface state, which leads to a separation of the CEC in two circles with opposite spin rotation direction [Figs. 3(a) and 3(b)]. The spin is aligned in plane and tangentially with the circular CEC, the outer circle (red) rotating counterclockwise and the inner circle (green) rotating clockwise. The two split surface state bands are described by the following equation [3]:

$$
\begin{aligned}
E\left(k_{\|}\right) & =\frac{\hbar^{2} k_{\|}^{2}}{2 m^{*}} \pm \gamma\left|k_{\|}\right|+E_{0}^{\prime} \\
& =\frac{\hbar^{2}}{2 m^{*}}\left(k_{\|} \pm k_{\mathrm{SOC}}\right)^{2}+E_{0},
\end{aligned}
$$

with $E_{0}^{\prime}=E_{0}+\hbar^{2} k_{\text {SOC }}^{2} / 2 m^{*}$. The latter equation describes two parabolic bands symmetrically shifted away from $k_{\|}=0$ with their extrema separated by $2 k_{\text {SOC }}$. A SOC strength of $\gamma=$ $396 \mathrm{meV} \AA$ was determined experimentally for the $\mathrm{Au}(111)$ surface by Henk et al. [2] from spin-resolved photoelectron spectroscopy data. This value corresponds to a splitting of the surface state parabolas of $2 k_{\mathrm{SOC}}=\frac{2 \gamma}{\hbar^{2}} m^{*}=0.26 \mathrm{~nm}^{-1}$ calculated for the surface state underneath the present graphene flake $\left(E_{0, \mathrm{G} / \mathrm{Au}}=-330 \mathrm{meV}\right.$ determined from STS spectra, $m^{*}=0.25 m_{e}$ from the fit with $\left.E=\hbar^{2} k_{\|}^{2} / 2 m^{*}+E_{0, \mathrm{G} / \mathrm{Au}}\right)$.

Revisiting scattering processes with the Rashba splitting in mind [Figs. 3(b) and 3(c)], one finds that for the $\mathrm{Au}(111)$ surface state a single ringlike feature centered at $\vec{q}=0$ is expected in FFTs due to a suppression of backscattering events for electrons between states with antiparallel spin orientation [21-24]. Hence only scattering between opposite points on two different circular $\mathrm{Au}$ CECs appears in the experiment. The observed ringlike feature in FT-LDOS is then produced by the scattering vectors $\vec{q}_{\mathrm{Au}, 2-\mathrm{Au}, 1}=\left(\vec{k}_{\mathrm{Au}, 1}-\vec{k}_{\mathrm{Au}, 2}\right)$ [large central ring in Fig. 2(d) and blue ring in Fig. 3(c)]. Vectors $\vec{k}_{\mathrm{Au}, 1}=\vec{k}_{\|}-\vec{k}_{\mathrm{SOC}}$ and $\vec{k}_{\mathrm{Au}, 2}=-\left(\vec{k}_{\|}+\vec{k}_{\mathrm{SOC}}\right)$ refer to the two circular CECs of Au. The measured data points of the scattering within the $\mathrm{Au}(111)$ surfaces state (blue rhombi), the parabolic fit to the data (blue solid curve), and the calculated dispersions of the spin-split bands (dash-dotted curves) are shown in Fig. 4.

The scattering vectors corresponding to the transitions between graphene states at the $K$ and $K^{\prime}$ points and the $\mathrm{Au}(111)$ surface state at the $\Gamma$ point [Figs. 3(b) and 3(c)] can be expressed in the general form as follows: $\vec{q}_{\mathrm{G}-\mathrm{Au}, 1 / 2}=$ $\left(\overrightarrow{\Gamma K}+\vec{k}_{\mathrm{G}}\right)-\vec{k}_{\mathrm{Au}, 1 / 2}$. Within the JDOS approximation we find that the resulting scattering vectors point at the region around the $\mathrm{BZ}$ corner which is limited by an inner circle with radius $\left|\vec{q}_{\mathrm{G}-\mathrm{Au}, 1 / 2}-\overrightarrow{\Gamma K}\right|=k_{\mathrm{Au}, 1 / 2}-k_{\mathrm{G}}$ and an outer circle with radius $\left|\vec{q}_{\mathrm{G}-\mathrm{Au}, 1 / 2}-\overrightarrow{\Gamma K}\right|=k_{\mathrm{Au}, 1 / 2}+k_{\mathrm{G}}$, for each Au surface state branch, respectively. At this point we have to consider the crystal momentum rather than the electron momentum and following the stationary phase approximation [20] one finds that quasiparticles will interfere constructively if the 
(a)

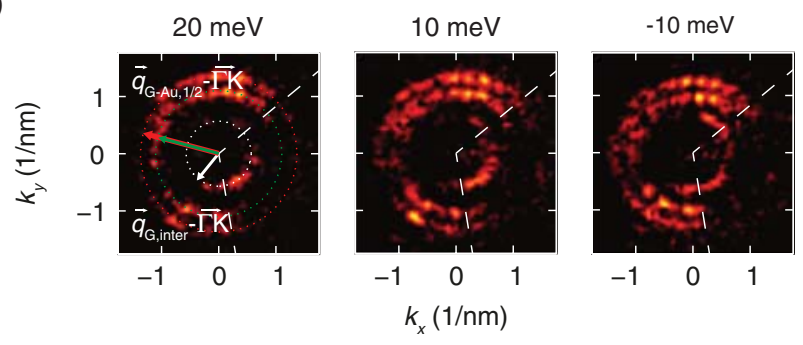

(b)

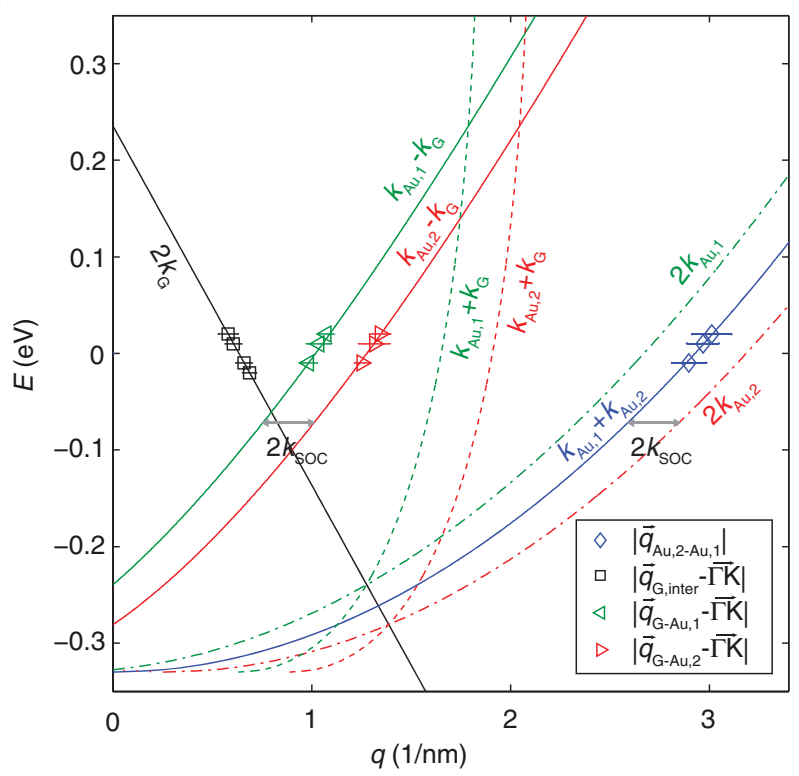

FIG. 4. (Color online) (a) Magnifications of the graphene and graphene-Au scattering features at the $\mathrm{BZ}$ corner $\left(3.5 \times 3.5 \mathrm{~nm}^{-2}\right)$ for various energies. Graphene-Au scattering vectors $\vec{q}_{\mathrm{G}-\mathrm{Au}, 1 / 2}$ are indicated (red and green arrows). (b) Energy dependence of the Au surface state backscattering feature around the FFT center (blue rhombi) as well as the graphene (black squares) and graphene-Au (red and green triangles) scattering features derived from two of the three BZ corners. The data points represent the average value of comparable FFT features. The blue curve represents a parabolic fit to the experimental points of the $\mathrm{Au}(111)$ surface state backscattering with the band minimum value obtained from STS. The dispersions of both spin-split branches of the $\mathrm{Au}(111)$ surface state are calculated from the experimental Au surface state scattering using the SOC strength $\gamma$ from Ref. [2] and plotted in dash-dotted lines. The dispersion of graphene-Au scattering vectors is calculated according to the equations in the text. Energy dependencies of the experimentally accessible scattering vectors are plotted in full lines and of not accessible scattering vectors in dashed lines.

group velocity $\vec{v}=\hbar^{-1} \nabla_{\vec{k}} E(\vec{k})$ is antiparallel for initial and final states [24,36,37]. Due to the $p$ doping of graphene on $\mathrm{Au}(111)$, i.e., with hole-like graphene bands and electron-like surface state bands, at energies close to $E_{\mathrm{F}}$ we can only expect scattering for the parallel alignment of $\vec{k}_{\mathrm{G}}$ and $\vec{k}_{\mathrm{Au}, 1 / 2}$. Consequently, only those $\vec{q}_{\mathrm{G}-\mathrm{Au}, 1 / 2}$ scattering vectors can be observed that form two additional rings with the radii $\left|\vec{q}_{\mathrm{G}-\mathrm{Au}, 1 / 2}-\overrightarrow{\Gamma K}\right|=k_{\mathrm{Au}, 1 / 2}-k_{\mathrm{G}}$ in addition to the intervalley scattering ring.

In Fig. 4(a) magnifications of one set of these additional scattering features at the BZ corners are shown for the three different energies close to $E_{\mathrm{F}}$. The determination of the radius $|\vec{q}-\overrightarrow{\Gamma K}|$ was carried out after an azimuthal integration of the FFT intensity around the center of the circles. In Fig. 4(b) the radii of the graphene, graphene- $\mathrm{Au}$, and $\mathrm{Au}$ scattering features are plotted versus energy. Regarding the graphene dispersion, the linear fit to the intervalley scattering data points yields similar fit parameters $\left[E_{\mathrm{D}}=(0.235 \pm 0.050) \mathrm{eV}, v_{\mathrm{F}}=(1.13 \pm\right.$ $0.2) \times 10^{6} \mathrm{~m} / \mathrm{s}$ ] as obtained in our earlier experiments [26]. Considering a splitting of the Au surface state bands of $2 k_{\text {SOC }}=0.26 \mathrm{~nm}^{-1}$ as derived above using published data [2], we first plot the energy dependence of the $\left|\vec{q}_{\mathrm{Au}, 1 / 2}\right|=2 k_{\mathrm{Au}, 1 / 2}$ scattering vectors for both branches of the spin-split $\mathrm{Au}(111)$ surface state [dash-dotted lines in Fig. 4(b)]. We further plot the energy dependence of the graphene-Au scattering features $\vec{q}_{\mathrm{G}-\mathrm{Au}, 1 / 2}$ for both the parallel and antiparallel orientation between $\vec{k}_{\mathrm{Au}}$ and $\vec{k}_{\mathrm{G}}$ (full and dashed red and green lines). The experimentally obtained graphene-Au scattering vectors (red and green triangles) coincide with the calculated energy dependence of the $\vec{q}_{\mathrm{G}-\mathrm{Au}, 1 / 2}$ vectors assuming the parallel alignment of $\vec{k}_{\mathrm{G}}$ and $\vec{k}_{\mathrm{Au}}$, thus perfectly reproducing the band separation and dispersion, which stems from the Rashba splitting of the $\mathrm{Au}(111)$ surface state. Moreover, the presented analysis shows that an easy access to the Rashba splitting of the metal surface state is given using the difference in radius of the two additional ringlike graphene-Au scattering features: $\Delta q=\left|\vec{q}_{\mathrm{G}-\mathrm{Au}, 2}-\vec{q}_{\mathrm{G}-\mathrm{Au}, 1}\right|=\left(k_{\mathrm{Au}, 2}-k_{\mathrm{G}}\right)-$ $\left(k_{\mathrm{Au}, 1}-k_{\mathrm{G}}\right)=2 k_{\mathrm{SOC}}$. Our measurements further demonstrate that although graphene on $\mathrm{Au}(111)$ is regarded to be quasifreestanding, a residual interaction between both materials is present allowing interband scattering processes between graphene and $\mathrm{Au}$ of considerable intensity, as observed in the FFT of the interference patterns.

Throughout our discussion we assumed that graphene bands are spin-degenerate. In the following we will discuss the impact of a possible spin texture of graphene [38] due to extrinsic SOC $[12,14]$ on the scattering at the graphene/Au(111) interface. In this case, the spin texture in graphene shows a spin orientation perpendicular to the momentum $\vec{k}_{\mathrm{G}}$ similar to the Rashba-split Au surface state, where the spin orientation is perpendicular to $\vec{k}_{\mathrm{Au}, 1 / 2}$. Since the spin orientations of graphene and Au are both locked perpendicular to their respective $\vec{k}$ vectors, transitions will only be allowed between the circular CECs with comparable spin-rotation direction. If we consider the experimentally observed doping, the Fermi level cuts both branches of the Rashba-split graphene cone in the graphene/Au(111) system. The same number of scattering features is expected with included Rashba splitting of the graphene states; however the radius of the graphene-Au scattering features will now be $\left|k_{\mathrm{Au}, 1 / 2}-k_{\mathrm{G}} \pm k_{\mathrm{SOC}, \mathrm{G}}\right|$ and thus enlarged due to the Rashba splitting in graphene if an inverted sense of rotation of the graphene spin texture is assumed according to $[3,39]$. The difference between the radii of the two additional ring features can be quantified to $\Delta q=\left|\vec{q}_{\mathrm{G}-\mathrm{Au}, 2}-\vec{q}_{\mathrm{G}-\mathrm{Au}, 1}\right|=$ $\left[k_{\mathrm{Au}, 2}-\left(k_{\mathrm{G}}-k_{\mathrm{SOC}, \mathrm{G}}\right)\right]-\left[k_{\mathrm{Au}, 1}-\left(k_{\mathrm{G}}+k_{\mathrm{SOC}, \mathrm{G}}\right)\right]=2 k_{\mathrm{SOC}, \mathrm{Au}}+$ $2 k_{\mathrm{SOC}, \mathrm{G}}$. In the case of a small splitting of $\Delta E_{\mathrm{SOC}, \mathrm{G}} \simeq$ $10 \mathrm{meV}$ [12], the radii of the scattering features will be undetectable within the experimental $k$ resolution, since the deviation from non-Rashba-split graphene will be on the 
order of $2 k_{\mathrm{SOC}, \mathrm{G}}=\Delta E_{\mathrm{SOC}, \mathrm{G}} / \hbar v_{F} \simeq 0.015 \mathrm{~nm}^{-1}$ compared to a splitting of the two graphene-Au scattering rings of $2 k_{\mathrm{SOC}, \mathrm{Au}}=0.26 \mathrm{~nm}^{-1}$. However, a giant Rashba splitting of $100 \mathrm{meV}$ as reported in [14] can be ruled out for the epitaxial graphene/Au(111) system studied here, since then $2 k_{\mathrm{SOC}, \mathrm{G}}$ would be roughly one half of the Au Rashba effect contribution $2 k_{\mathrm{SOC}, \mathrm{Au}}$, which we would have been able to observe.

In conclusion, our investigations on the graphene/Au(111) system employing quasiparticle interference mapping show that using graphene bands as an additional channel for scattering, one can visualize the Rashba-split surface state of $\mathrm{Au}(111)$ by means of a local probe. We find that FFT features attributed to the interband scattering processes involving both graphene and the Rashba-split Au(111) surface states preserve a spin-split signature unlike the observed surface state backscattering on pure Au surfaces, which does not provide information about the spin splitting. The measurement of the spin-split states at the nanometer scale in epitaxial graphene/metal systems opens a new possible approach to study the band structure of surfaces with spin-orbit coupling.

We thank G. Bihlmayer for helpful discussions. This work was supported by the Deutsche Forschungsgemeinschaft (DFG) within the Priority Program (SPP) 1459 (Grant No. FO 640/4-1) and by the European Science Foundation under the EUROCORES Programme EuroGRAPHENE (Project SpinGraph) funded through the DFG (Grant No. FO 640/2-1).
[1] S. LaShell, B. A. McDougall, and E. Jensen, Phys. Rev. Lett. 77, 3419 (1996).

[2] J. Henk, M. Hoesch, J. Osterwalder, A. Ernst, and P. Bruno, J. Phys.: Condens. Matter 16, 7581 (2004).

[3] M. Hoesch, M. Muntwiler, V. N. Petrov, M. Hengsberger, L. Patthey, M. Shi, M. Falub, T. Greber, and J. Osterwalder, Phys. Rev. B 69, 241401 (2004).

[4] G. Jezequel, Y. Petroff, R. Pinchaux, and F. Yndurain, Phys. Rev. B 33, 4352 (1986).

[5] T. Hirahara, K. Miyamoto, I. Matsuda, T. Kadono, A. Kimura, T. Nagao, G. Bihlmayer, E. Chulkov, S. Qiao, K. Shimada, H. Namatame, M. Taniguchi, and S. Hasegawa, Phys. Rev. B 76, 153305 (2007).

[6] K. Sugawara, T. Sato, S. Souma, T. Takahashi, M. Arai, and T. Sasaki, Phys. Rev. Lett. 96, 046411 (2006).

[7] Yu. A. Bychkov and E. I. Rashba, JETP Lett. 39, 78 (1984).

[8] C. R. Ast, J. Henk, A. Ernst, L. Moreschini, M. C. Falub, D. Pacilé, P. Bruno, K. Kern, and M. Grioni, Phys. Rev. Lett. 98, 186807 (2007).

[9] C. R. Ast, G. Wittich, P. Wahl, R. Vogelgesang, D. Pacilé, M. C. Falub, L. Moreschini, M. Papagno, M. Grioni, and K. Kern, Phys. Rev. B 75, 201401 (2007).

[10] D. Pacilé, C. R. Ast, M. Papagno, C. Da Silva, L. Moreschini, M. Falub, A. P. Seitsonen, and M. Grioni, Phys. Rev. B 73, 245429 (2006).

[11] L. Moreschini, A. Bendounan, I. Gierz, C. R. Ast, H. Mirhosseini, H. Höchst, K. Kern, J. Henk, A. Ernst, S. Ostanin, F. Reinert, and M. Grioni, Phys. Rev. B 79, 075424 (2009).

[12] A. Varykhalov, J. Sánchez-Barriga, A. M. Shikin, C. Biswas, E. Vescovo, A. Rybkin, D. Marchenko, and O. Rader, Phys. Rev. Lett. 101, 157601 (2008).

[13] D. Ma, Z. Li, and Z. Yang, Carbon 50, 297 (2012).

[14] D. Marchenko, A. Varykhalov, M. Scholz, G. Bihlmayer, E. Rashba, A. Rybkin, A. Shikin, and O. Rader, Nat. Commun. 3, 1232 (2012).

[15] Y. Yao, F. Ye, X.-L. Qi, S.-C. Zhang, and Z. Fang, Phys. Rev. B 75, 041401 (2007).

[16] J. C. Boettger and S. B. Trickey, Phys. Rev. B 75, 121402 (2007).

[17] M. Gmitra, S. Konschuh, C. Ertler, C. Ambrosch-Draxl, and J. Fabian, Phys. Rev. B 80, 235431 (2009).

[18] J. E. Hoffman, K. McElroy, D.-H. Lee, K. M. Lang, H. Eisaki, S. Uchida, and J. C. Davis, Science 297, 1148 (2002).

[19] L. Simon, F. Vonau, and D. Aubel, J. Phys.: Condens. Matter 19, 355009 (2007).
[20] L. Simon, C. Bena, F. Vonau, M. Cranney, and D. Aubel, J. Phys. D: Appl. Phys. 44, 464010 (2011).

[21] L. Petersen and P. Hedegård, Surf. Sci. 459, 49 (2000).

[22] J. I. Pascual, G. Bihlmayer, Yu. M. Koroteev, H.-P. Rust, G. Ceballos, M. Hansmann, K. Horn, E. V. Chulkov, S. Blügel, P. M. Echenique, and Ph. Hofmann, Phys. Rev. Lett. 93, 196802 (2004).

[23] P. Roushan, J. Seo, C. V. Parker, Y. S. Hor, D. Hsieh, D. Qian, A. Richardella, M. Z. Hasan, R. J. Cava, and A. Yazdani, Nature (London) 460, 1106 (2009).

[24] L. El-Kareh, P. Sessi, T. Bathon, and M. Bode, Phys. Rev. Lett. 110, 176803 (2013).

[25] M. Steinbrecher, H. Harutyunyan, C. R. Ast, and D. Wegner, Phys. Rev. B 87, 245436 (2013).

[26] P. Leicht, L. Zielke, S. Bouvron, R. Moroni, E. Voloshina, L. Hammerschmidt, Yu. S. Dedkov, and M. Fonin, ACS Nano 8, 3735 (2014).

[27] J. Coraux, A. T. N'Diaye, M. Engler, C. Busse, D. Wall, N. Buckanie, F.-J. Meyer zu Heringdorf, R. van Gastel, B. Poelsema, and T. Michely, New J. Phys. 11, 023006 (2009).

[28] G. M. Rutter, J. N. Crain, N. P. Guisinger, T. Li, P. N. First, and J. A. Stroscio, Science 317, 219 (2007).

[29] I. Brihuega, P. Mallet, C. Bena, S. Bose, C. Michaelis, L. Vitali, F. Varchon, L. Magaud, K. Kern, and J. Veuillen, Phys. Rev. Lett. 101, 206802 (2008).

[30] P. Mallet, I. Brihuega, S. Bose, M. M. Ugeda, J. M. GómezRodríguez, K. Kern, and J. Y. Veuillen, Phys. Rev. B 86, 045444 (2012).

[31] C. Bena, Phys. Rev. Lett. 100, 076601 (2008).

[32] A. Bergvall and T. Löfwander, Phys. Rev. B 87, 205431 (2013).

[33] P. Avouris, J. Vac. Sci. Tech. B 12, 1447 (1994).

[34] K. Schouteden, P. Lievens, and C. Van Haesendonck, Phys. Rev. B 79, 195409 (2009).

[35] S. J. Altenburg, J. Kröger, T. O. Wehling, B. Sachs, A. I. Lichtenstein, and R. Berndt, Phys. Rev. Lett. 108, 206805 (2012).

[36] L. M. Roth, H. J. Zeiger, and T. A. Kaplan, Phys. Rev. 149, 519 (1966).

[37] Q. Liu, X.-L. Qi, and S.-C. Zhang, Phys. Rev. B 85, 125314 (2012).

[38] F. Kuemmeth and E. I. Rashba, Phys. Rev. B 80, 241409 (2009).

[39] E. I. Rashba, Phys. Rev. B 79, 161409 (2009). 